\title{
Terbutaline in the treatment of preterm labor: A comparison of intravenous and subcutaneous administration
}

\author{
LYNNE HASPEDIS, DO \\ DAVID WOLF, DO
}

\begin{abstract}
Experience with two routes of terbutaline administration was reviewed to compare their effectiveness in preventing premature labor. Of the 70 patients receiving terbutaline sulfate therapy, 55 patients were placed on a subcutaneous protocol and 15 patients were on an intravenous protocol. Both groups received oral terbutaline therapy after contractions had ceased. Results were assessed in terms of duration of therapy and Richter's prolongation index. Statistical analysis showed no significant difference between the two groups in regard to effectiveness of tocolysis or in frequency of maternal side effects. Choice of terbutaline delivery route is considered in the light of the reported potential for complications.
\end{abstract}

Preterm delivery remains a major factor contributing to perinatal mortality. A wide variety of methods for inhibiting preterm labor have been used: bedrest, prophylactic progesterone, ethanol, and most recently, $\beta$-mimetic agents such as ritodrine hydrochloride and terbutaline sulfate. $B$ sympathomimetics occupy a central position in the treatment of premature labor. Attempts have been made to develop tocolytic agents that will effectively inhibit premature uterine contractions with the fewest metabolic and cardiovascular side effects.

The administration of sympathomimetic drugs usually has been initiated by the intravenous (IV) $i$ route. The infusion rate at first is low then increased gradually until cessation of labor; a maintenance dose is given for several hours before the start of oral therapy. As Stubblefield and Heyl ${ }^{1}$ have shown, IV therapy has an obvious advantage: one can titrate the dose and reduce it immediately in the event of an adverse response. However, they point out that maternal metabolic changes including antidiuresis and water retention, hypokalemia and ketoacidosis, and hyperglycemia have occurred during the first phase of IV treatment. In addition, the use of an initial low-dose infusion rate that is gradually increased could allow cervical dilation to progress before the effective dosage is reached.

Under the IV protocol as used at Riverside Osteopathic Hospital, the infusion rate of terbuta -line results in a dose of $0.49 \mathrm{mg}$ to $0.73 \mathrm{mg}$ during the first hour of treatment; the usual subcutaneous dose, however, would be only $0.25 \mathrm{mg} / \mathrm{h}$. Stubblefield and Heyl ${ }^{1}$ have theorized that if subcutaneous and IV routes were equally effective, subcutaneous administration might be preferable. Our retrospective study was designed to compare the efficacy of intravenous terbutaline with that of subcutaneous terbutaline in the treatment of premature labor.

\section{Materials and methods}

The population comprised all patients receiving terbutaline therapy over a 2-year-period from January 1984 through December 1985 at Riverside Osteopathic Hospital. During this time, 2,234 patients gave birth. Of these, 70 patients ( 73 pregnancies) $(\mathrm{N}=3)(3 \%)$ were treated with terbutaline. They all had singleton pregnancies with a diagnosis of labor prior to 36 weeks' gestation. The same patient was included in the study twice if during the time of the study she underwent more than one pregnancy complicated by premature labor requir- 
ing terbutaline therapy.

The assessment of gestational age and the diagnosis of premature labor was made by the resident physician in charge of the labor floor at the time the patient was admitted. Evaluation included careful history and physical examination, review of prenatal records, and evaluation of ultrasound reports and external fetal monitor (EFM) patterns. The most important objective criteria considered were regular uterine contractions (every 3 to 6 minutes) as recorded by EFM and a change in the condition of the cervix during the initial evaluation period.

Uterine contractions were recorded by external tocography, and fetal heart rate was monitored by external ultrasound technique. After the diagnosis of premature labor was confirmed, an additional 15-minute baseline recording was obtained to document uterine activity and fetal heart rate before therapy was started. Laboratory evaluation included CBC, urinalysis, electrolyte determination, blood glucose test, and urine and vaginal cultures.

Patients were not considered candidates for terbutaline administration if one or more of the following conditions were present at the time of initial evaluation: abruptio placentae, fetal abnormality, premature rupture of membranes, maternal arrhythmia, hypertension, hyperthyroidism, or diabetes. Informed consent was obtained from each patient before terbutaline therapy was begun.

\section{Intravenous terbutaline protocol}

An intravenous infusion of $1,000 \mathrm{~mL}$ lactated Ringer's solution was started at a keep-open rate. Terbutaline sulfate $4 \mathrm{mg}$ was added to $1,000 \mathrm{~mL}$ lactated Ringer's solution and this was administered by IVAC infusion pump via piggyback with the hydration solution. A bolus of $0.25 \mathrm{mg}$ terbutaline was first given intravenously over a 5-minute time span. Immediately afterward the infusion of terbutaline was begun at the rate of $0.004 \mathrm{mg} /$ min. Contractions and fetal heart tones were observed and recorded on the EFM throughout treatment. If no change in frequency, duration, or intensity of contractions was noted for ten minutes, the infusion rate was increased by $0.004 \mathrm{mg} / \mathrm{min}$. every five minutes to a maximum of $0.016 \mathrm{mg} /$ min. If contractions began to subside in number and frequency, the infusion rate was maintained for a minimum of one hour. Once contractions ceased or greatly subsided for at least one hour, the infusion was stopped. A subcutaneous dose of $0.25 \mathrm{mg}$ terbutaline was given at the time infusion was discontinued and every 3 to 4 hours thereafter. If contractions remained absent after 24 hours, oral terbutaline, $2.5 \mathrm{mg}$ to $5.0 \mathrm{mg}$ every 4 to 6 hours, was given.
Vital signs including blood pressure and heart rate, fetal heart tones, and behavior (affect) were noted three times over a 15-minute-period prior to treatment for baseline values and then every 15 minutes during terbutaline infusion. Once subcutaneous therapy was begun, vital signs were taken every four hours and, for oral therapy, every shift.

The following side effects were observed by the nursing staff and brought to the attention of the resident staff: (1) maternal tachycardia or ectopic beats; (2) hypotension; (3) muscular tremors; (4) inappropriate behavior; (5) flushing; and (6) fetal tachycardia.

\section{Subcutaneous terbutaline protocol}

An infusion of 1,000 mL lactated Ringer's solution was started at a rate of $125 \mathrm{~mL} / \mathrm{h}$. Terbutaline was then injected subcutaneously into the deltoid region at a dose of $0.25 \mathrm{mg}$. A subcutaneous dose was given hourly for a total of three doses, then every three to four hours thereafter. Contractions and fetal heart tones were observed and recorded continuously on the EFM. If contractions were absent after 24 hours of therapy, the patient then was given oral terbutaline at the dosage of $2.5 \mathrm{mg}$ to $5.0 \mathrm{mg}$ every 4 to 6 hours. The oral dose was given at the same time as the last subcutaneous dose.

The vital signs and blood pressure, heart rate, fetal heart tones, and behavior (affect) were observed as with intravenous therapy, except that they were noted every four hours during subcutaneous administration. The same side effects noted in the IV protocol were noted by the nursing staff and brought to the attention of the resident.

Patients continued with oral terbutaline therapy until they completed 37 weeks' gestation. Patients who were electively taken off terbutaline therapy or who chose to discontinue it were deleted from the study.

\section{Analysis of results}

All charts and labor records of the patients as well as neonatal charts of the delivered infants were reviewed. Evaluation of success of each method of tocolysis was obtained by using two different measurements. The number of days gained in utero, calculated from the time of onset of therapy to delivery date (in weeks), with success defined as $>37$ weeks at delivery, was termed the "duration of therapy." A more effective method of evaluating tocolytic success, the Prolongation Index (PI) as developed by Richter ${ }^{2}$, was also used to evaluate the success of tocolysis in each group. The PI equals 100 times the value of the lag time between the start of tocolytic therapy and delivery, divided by the gestational age at admission. The PI value in- 
creases as lag time increases; however, the farther pregnancy is advanced at the start of tocolytic therapy, the lower the PI value.

A PI of 8 but less than 50 corresponds to a lag time of more than two weeks but gestational age of $<38$ weeks and suggests incomplete success. Scores greater than 50 correspond to a lag time of more than two weeks and gestational age at delivery of $>37$ weeks; such scores indicate full success. Failure is shown by a score of less than 8 with a lag time of less than two weeks.

Parity, the gestational age and degree of cervical effacement, and a maximum cervical dilation at onset of therapy, gestational age at delivery, route of delivery, fetal distress, birth weight of the neonates, Apgar score, and number of maternal readmissions to the hospital also were analyzed in relation to the outcome of tocolysis. Statistical analysis was performed with both the Student's $T$ test and $\mathrm{X}^{2}$ analysis. Probability values of $P<.05$ were taken to represent statistically significant findings.

\section{Results}

The 70 patients were randomly divided into two groups by a resident on call. Group 1 consisted of 55 patients $(79 \%)$ placed on the subcutaneous protocol and group 2 consisted of 15 patients (25\%) placed on the IV protocol.

The mean duration of therapy for group 1 was 7.1 weeks and for group 2, 6.6 weeks. The difference between the two groups was not statistically significant. For the total study population the minimum duration of therapy was 1 week ( 3 patients from group 1 and 1 from group 2). The maximum duration of therapy was 21 weeks, in a patient who began subcutaneous terbutaline therapy at 21 weeks' gestation and gave birth at 42 weeks' gestation.

The mean PI value for both groups was 22 (group 1, 22.3; group 2, 22.9). According to Richter's ${ }^{2}$ study, these values correspond to a lag time of $>$ 2 weeks, so the results are categorized as incompletely successful. Only six patients had a PI score $>50$. Of these six, five received terbutaline by the subcutaneous route and 1 , by IV. This may suggest a much higher success rate with subcutaneous terbutaline; however, the number of patients is too small to be statistically significant. PI values $<8$ suggest failure; such values were shown by nine patients. Of those 9 in whom tocolytic therapy failed, six received subcutaneous terbutaline and three, IV terbutaline. Again the distribution of patients in the study groups prevents demonstration of a statistically significant difference between groups 1 and 2 in terms of failed tocolysis.

Gestational age at onset of tocolytic therapy was signficantly different in group 1 and group 2 . The mean gestational age at onset of treatment was 32 weeks in group 1 and 30 weeks in group 2. $(P$ $=.029)$. This result would suggest a bias toward selection of IV terbutaline by the resident staff for those patients with premature labor at a lower gestational age. However, neither the PI nor durationof-therapy methods of assessment showed any relation between success of tocolytic therapy and gestational age at the start of therapy.

The mean parity for both groups was 1.0. There was no apparent statistically significant relationship between the patient's parity and the success of therapy in either group 1 or 2 .

The patients were given cervical effacement values of $0 \%, 50 \%$, or $100 \%$ at the start of tocolysis. In the majority of pregnancies (57), cervical effacement was $0 \%$ at the time of diagnosis of premature labor; in 14 , it was $50 \%$ and in 2 , it was $100 \%$. The cervical effacement values did not appear to make a statistically significant difference in the method of tocolysis chosen or the success of the tocolysis route used. The mean PI value for patients with $0 \%$ effacement in group 1 was 23.8 , in group $2,23.2$. The duration of therapy (weeks) for patients with $0 \%$ effacement in group 1 was 7.1 weeks; in group $2,6.6$ weeks. If effacement was judged as $50 \%$ or greater there still appeared to be no significant difference in PI value or duration of therapy between group 1 and 2.

The maximum cervical dilation prior to onset of tocolysis was $4 \mathrm{~cm}$. Two patients had $4 \mathrm{~cm}$ dilation at the onset of tocolysis. One was at 30 weeks' gestation and the decision was made to use IV tocolysis, which was "fully successful" with delivery at 40 weeks' gestation. The other patient was at 35 weeks' gestation at the time tocolysis was begun and subcutaneous terbutaline was used. She was subsequently delivered of an infant at 37 weeks. The PI value for this patient was only 6.0 , suggesting failure of therapy. The cervical dilation of other pregnancies $(\mathrm{N}=73)$ in the study varied from closed cervix $(\mathrm{N}=58)$ to $2 \mathrm{~cm}(\mathrm{~N}=9)$ to 3 $\mathrm{cm}(\mathrm{N}=4)$. No statistically significant relationship was seen between cervical dilation and the success of IV or subcutaneous tocolysis in prolonging gestation.

The mean gestational age at delivery was slightly higher in group 1 (group $1,38.2$ years; group 2, 36.7 years), however, this difference was not statistically significant $(P=.516)$. The minimum gestational age at delivery was 22 weeks and was associated with fetal death thought to be secondary to placental abruption. The terbutaline was initially given by IV. The maximum gestational age at delivery was 43 weeks $(N=2)$. Both pa- 
tients who gave birth at 43 weeks were in group 1. A total of 5 pregnancies involved delivery at 42 weeks or more. All these postdate patients were in group 1 . Of these 5,3 required induction of labor for postdatism, 2 gave birth vaginally, and 1 underwent primary cesarean section after two days' induction of labor failed.

Among the 73 pregnancies in the study group, there were 50 vaginal deliveries and 23 cesarean sections, of which 9 were primary cesarean section ( $12 \%$ rate). No statistically significant difference was found between the subcutaneous and IV group in regard to route of delivery.

Fetal or neonatal distress (defined as intrapartum distress diagnosed on EFM tracings, neonatal distress requiring resuscitation, or neonatal respiratory distress syndrome [RDS]) was evaluated in both groups of patients. There were ten patients whose infant experienced intrapartum distress. Of these ten, six were from group 1 (intrauterine growth retardation, 2; postmature syndrome, 1; placental abruption, 1; RDS, 1; meconium aspiration, 1 ); and 1, who demonstrated placental abruption, was from group 2 . In the remaining three, the cause of intrapartum distress was undetermined. Fetal distress was diagnosed for only one patient while she was receiving tocolytic therapy. This was the group 2 patient with the 22 weeks' gestation terminating in placental abruption and fetal death.

The mean birth weight in group 1 was $3,187 \mathrm{~g}$ and in group $2,3,1818 \mathrm{~g}$. The difference was not significant. There were six macrosomic infants $(>$ $4,000 \mathrm{~g}$ ), all from group 1.

The incidence of low Apgar scores, defined as 5 -minute score $<6$, was not statistically different in the two groups. Only two infants had a 5-min111 ute score $<6$. One was the infant who died at 22 weeks because of placental abruption (group 2). The other infant had a 5-minute Apgar score of 5 . This also was a case of placental abruption. The mother had subcutaneous tocolysis until week 37 and gave birth at week 40 .

The greatest number of readmissions to the hospital for any one gestation was three. Two patients were readmitted to the hospital for a total of three times, each after the initial start of terbutaline therapy and prior to the delivery admission. Two of these patients were from group 2, the other from group 1. Readmissions were due to recurrence of premature labor. The difference between the IV and subcutaneous terbutaline groups in terms of number of readmissions to the hospital was not significantly different. The majority of patients $(\mathrm{N}=$ 50) from both groups required no readmission to the hospital after the initial beginning of tocolytic therapy until the admission for delivery.
Maternal side effects encountered consisted of hypokalemia $(\mathrm{K}+<3.2)$, hyperglycemia, emesis, severe anxiety, severe tremors, and behavioral abnormalities requiring discontinuation or change in therapy. Only 29 patients reported significantly disturbing side effects. Of these one required discontinuation of therapy secondary to extreme anxiety and possible seizure activity while being given subcutaneous terbutaline. The predominant side effect, seen in 22 patients, was hypokalemia. There was no significant difference between the two groups in the frequency of side effects.

\section{Discussion}

Various methods have been selected to determine the success of tocolytic therapy. A recent assessment of these compared the TI (tocolytic index), PI, Y-intercept, Weidinger score with the simple criterion of delivery at 37 weeks showed that the latter method was adequate for comparing the success of different agents. ${ }^{3}$ As noted by Richter, ${ }^{2}$ however, this method fails to show any benefit by increasing the time to delivery from week 27 , for example, to week 36 , in spite of the improved chances for fetal survival at 36 weeks. The PI index also takes into account differences in gestational age at the onset of therapy. Therefore, in our study, both the clinical definition of successful tocolysis (gestational age at delivery $>37$ weeks) and Richter's PI were used to evaluate tocolytic success.

A number of interesting trends developed in the comparison of routes of administration of terbutaline tocolysis. Both IV and subcutaneous routes were equally successful in terms of duration of therapy, since both were associated with a lag time of $>2$ weeks. In terms of the PI, there was no greater success with the IV than with the subcutaneous therapy: both groups had very similar PI values. The data did reveal a trend toward higher PI values in the subcutaneous group; however, this trend could simply reflect the greater number of patients in the study population undergoing subcutaneous therapy.

The data showed a statistically significant difference between the two groups in gestational age at onset of therapy. The resident staff chose IV terbutaline therapy more often in patients at a lower gestational age, and since the success rate of the IV, terbutaline was equal to that of subcutaneous terbutaline, it could be assumed that IV therapy can work quickly at early gestation and be as effective as subcutaneous therapy.

Parity and degree of cervical effacement did not influence the success rate of tocolytic therapy. Cervical dilation values showed improved outcome in patients who underwent IV therapy; however, the 
numbers were too small to show any statistical significance. The data trend would suggest choosing IV therapy over subcutaneous therapy in patients with advanced cervical dilation.

Gestational age at delivery and route of delivery were similar for both groups. As expected, neither mode of tocolytic therapy was associated with a higher cesarean-section rate. Macrosomia did appear to predominate in the subcutaneous therapy group. All the postdate gestations also were among group 1 patients. Further evaluation of the relationship between subcutaneous terbutaline, macrosomia, and postdate gestation may elucidate this relationship.

In terms of fetal and neonatal distress there was no statistically significant difference between the two groups. The data suggested a trend toward an increased number of patients with fetal distress among the subcutaneous terbutaline groups; however, this trend may simply be due to an unevenly divided study population with a predominance of patients undergoing subcutaneous treatment.

Readmission to the hospital, as well as troublesome or dangerous side effects while on tocolytic therapy, are important parameters to evaluate in selection of a tocolytic agent and its route of administration. In general, the number of readmissions to the hospital for both groups was very low. The IV terbutaline group had a slightly higher number of readmissions; however, the difference was not statistically significant.

Adverse side effects were equally divided between both groups.

\section{Conclusions}

These data clearly demonstrate equally successful tocolysis with either subcutaneous or IV administration of terbutaline. Stubblefield and $\mathrm{Heyl}^{1}$ cite Leferink and co-workers' studies to show that ter- butaline is absorbed very rapidly after subcutaneous administration, the absorption half-life being only seven minutes. The serious adverse cardiovascular effects with $ß$-adrenergic tocolysis, such as maternal pulmonary edema and myocardial ischemia, usually have been associated with IV administration. In most cases these were diagnosed after the first 24 hours of therapy. ${ }^{4}$

We would like to propose that there may be no need for prolonged intravenous terbutaline use and that an equally successful and potentially less harmful method of administration of terbutaline tocolysis would be a combination of the two routes of administration. For those patients at an early gestational age about whom physicians are most anxious, we would recommend terbutaline $0.25 \mathrm{mg}$ IV push followed by subcutaneous terbutaline $0.25 \mathrm{mg}$ every hour for three doses and every three to four hours thereafter. For all other patients, initial therapy would consist of subcutaneous terbutaline every hour for three doses followed by a subcutaneous dose every 3 to 4 hours. Both groups would be placed on an oral terbutaline regimen at the appropriate time prior to discharge.

1. Stubblefield PG, Heyl PS: Treatment of premature labor with subcutaneous terbutaline. Obstet Gynecol 1982;59:457-462.

2. Richter R: Evaluation of success in treatment of threatening premature labor by betamimetic drugs. Am J Obstet Gynecol 1977;127:482486.

3. Gummerus M: Best criterion for the assessment of efficacy of tocolysis and choice of most suitable betasympathomimetic. Acta Obstet Gynecol Scan 1985;64:561-565.

4. Katz M, Robertson PA, Creasy RK: Cardiovascular complications associated with terbutaline treatment for preterm labor. Am J Obstet $G y$ necol 1981;139:605-608.

From the Department of Obstetrics and Gynecology, Riverside Osteopathic Hospital, Trenton, Michigan.

Dr Haspedis, 2865 West Rd, Trenton, MI 48183. 